Research Article

\title{
Some Results on an Infinite Family of Nonexpansive Mappings and an Inverse-Strongly Monotone Mapping in Hilbert Spaces
}

\author{
Peng Cheng ${ }^{1}$ and Anshen Zhang ${ }^{2}$ \\ ${ }^{1}$ School of Mathematics and Information Science, North China University of Water Resources and Electric \\ Power, Zhengzhou 450011, China \\ ${ }^{2}$ No. 13 Zhongxue, Feng-Feng Kuangqu, Hangdan 056000, China
}

Correspondence should be addressed to Peng Cheng, hschengp@163.com

Received 8 September 2012; Accepted 10 October 2012

Academic Editor: Xiaolong Qin

Copyright (C) 2012 P. Cheng and A. Zhang. This is an open access article distributed under the Creative Commons Attribution License, which permits unrestricted use, distribution, and reproduction in any medium, provided the original work is properly cited.

\begin{abstract}
We study the problem of approximating a common element in the common fixed point set of an infinite family of nonexpansive mappings and in the solution set of a variational inequality involving an inverse-strongly monotone mapping based on a viscosity approximation iterative method. Strong convergence theorems of common elements are established in the framework of Hilbert spaces.
\end{abstract}

\section{Introduction and Preliminaries}

Let $H$ be a real Hilbert space, whose inner product and norm are denoted by $\langle\cdot, \cdot\rangle$ and $\|\cdot\|$, respectively. Let $C$ be a nonempty, closed, and convex subset of $H$. Let $A: C \rightarrow H$ be a mapping. Let $P_{C}$ be the metric projection from $H$ onto the subset $C$. The classical variational inequality is to find $u \in C$ such that

$$
\langle A u, v-u\rangle \geq 0, \quad \forall v \in C
$$

In this paper, we use $\operatorname{VI}(C, A)$ to denote the solution set of the variational inequality. For a given point $z \in H, u \in C$ satisfies the inequality

$$
\langle u-z, v-u\rangle \geq 0, \quad \forall v \in C,
$$


if and only if $u=P_{C} z$. It is known that projection operator $P_{C}$ is nonexpansive. It is also know that $P_{C}$ satisfies

$$
\left\langle x-y, P_{C} x-P_{C} y\right\rangle \geq\left\|P_{C} x-P_{C} y\right\|^{2}, \quad \forall x, y \in H .
$$

One can see that the variational inequality (1.1) is equivalent to a fixed point problem. The point $u \in C$ is a solution of the variational inequality (1.1) if and only if $u \in C$ satisfies the relation $u=P_{C}(u-\lambda A u)$, where $\lambda>0$ is a constant.

Recall the following definitions.

(a) $A$ is said to be monotone if and only if

$$
\langle A x-A y, x-y\rangle \geq 0, \quad x, y \in C .
$$

(b) $A$ is said to be $\alpha$-strongly monotone if and only if there exists a positive real number $\alpha$ such that

$$
\langle A x-A y, x-y\rangle \geq \alpha\|x-y\|^{2}, \quad x, y \in C .
$$

(c) $A$ is said to be $\alpha$-inverse-strongly monotone if and only if there exists a positive real number $\alpha$ such that

$$
\langle A x-A y, x-y\rangle \geq \alpha\|A x-A y\|^{2}, \quad \forall x, y \in C .
$$

(d) A mapping $S: C \rightarrow C$ is said to be nonexpansive if and only if

$$
\|S x-S y\| \leq\|x-y\|, \quad \forall x, y \in C
$$

In this paper, we use $F(S)$ to denote the fixed point set of $S$.

(e) A mapping $f: C \rightarrow C$ is said to be a $\kappa$-contraction if and only if there exists a positive real number $\mathcal{\kappa} \in(0,1)$ such that

$$
\|f(x)-f(y)\| \leq \kappa\|x-y\|, \quad \forall x, y \in C
$$

(f) A linear bounded operator $B$ on $H$ is strongly positive if and only if there exists a positive real number $\bar{\gamma}$ such that

$$
\langle B x, x\rangle \geq \bar{\gamma}\|x\|^{2}, \quad \forall x \in H .
$$

(g) A set-valued mapping $T: H \rightarrow 2^{H}$ is called monotone if and only if for all $x, y \in$ $H, f \in T x$, and $g \in T y$ imply $\langle x-y, f-g\rangle \geq 0$. A monotone mapping $T: H \rightarrow 2^{H}$ is maximal if the graph of $G(T)$ of $T$ is not properly contained in the graph of any other monotone mapping. It is known that a monotone mapping $T$ is maximal if and only if for $(x, f) \in H \times H,\langle x-y, f-g\rangle \geq 0$ for every $(y, g) \in G(T)$ implies $f \in T x$. Let $A$ be a monotone 
map of $C$ into $H$ and let $N_{C} v$ be the normal cone to $C$ at $v \in C$, that is, $N_{C} v=\{w \in H$ : $\langle v-u, w\rangle \geq 0$, for all $u \in C\}$ and define

$$
T v= \begin{cases}A v+N_{C} v, & v \in C, \\ \emptyset, & v \notin C .\end{cases}
$$

Then $T$ is maximal monotone and $0 \in T v$ if and only if $v \in \operatorname{VI}(C, A)$; see [1] and the reference therein.

For finding a common element in the fixed point set of nonexpansive mappings and in the solution set of the variational inequality involving inverse-strongly mappings, Takahashi and Toyoda [2] introduced the following iterative process:

$$
x_{0} \in C, \quad x_{n+1}=\alpha_{n} x_{n}+\left(1-\alpha_{n}\right) S P_{C}\left(x_{n}-\lambda_{n} A x_{n}\right), \quad \forall n \geq 0,
$$

where $A$ is an $\alpha$-inverse-strongly monotone mapping, $\left\{\alpha_{n}\right\}$ is a real number sequence in $(0,1)$, and $\left\{\lambda_{n}\right\}$ is a real number sequence in $(0,2 \alpha)$. They showed that the sequence $\left\{x_{n}\right\}$ generated in (1.11) weakly converges to some point $z \in F(S) \cap \operatorname{VI}(C, A)$ provided that $F(S) \cap \operatorname{VI}(C, A)$ is nonempty.

In order to obtain a strong convergence theorem of common elements, Iiduka and Takahashi [3] considered the problem by the following iterative process:

$$
x_{0} \in C, \quad x_{n+1}=\alpha_{n} x+\left(1-\alpha_{n}\right) S P_{C}\left(x_{n}-\lambda_{n} A x_{n}\right), \quad \forall n \geq 0,
$$

where $x$ is a fixed element in $C, A$ is an $\alpha$-inverse-strongly monotone mapping, $\left\{\alpha_{n}\right\}$ is a real number sequence in $(0,1)$, and $\left\{\lambda_{n}\right\}$ is a real number sequence in $(0,2 \alpha)$. They showed that the sequence $\left\{x_{n}\right\}$ generated in (1.12) strongly converges to some point $z \in F(S) \cap \operatorname{VI}(C, A)$ provided that $F(S) \cap \mathrm{VI}(C, A)$ is nonempty.

Iterative methods for nonexpansive mappings have been applied to solve convex minimization problems; see, for example, [4-8] and the references therein. A typical problem is to minimize a quadratic function over the set of the fixed points a nonexpansive mapping $S$ on a real Hilbert space $H$ :

$$
\min _{x \in F(S)} \frac{1}{2}\langle B x, x\rangle-\langle x, b\rangle
$$

where $B$ is a linear bounded self-adjoint operator, and $b$ is a given point in $H$. In [4], it is proved that the sequence $\left\{x_{n}\right\}$ defined by the iterative method below, with the initial guess $x_{0} \in H$ chosen arbitrarily,

$$
x_{n+1}=\left(I-\alpha_{n} B\right) S x_{n}+\alpha_{n} b, \quad n \geq 0,
$$

strongly converges to the unique solution of the minimization problem (1.13) provided that the sequence $\left\{\alpha_{n}\right\}$ satisfies certain conditions. 
Recently, Marino and $\mathrm{Xu}$ [5] considered the problem by viscosity approximation method. They study the following iterative process:

$$
x_{0} \in C, \quad x_{n+1}=\left(I-\alpha_{n} B\right) S x_{n}+\alpha_{n} \gamma f\left(x_{n}\right), \quad n \geq 0,
$$

where $f$ is a contraction. They proved that the sequence $\left\{x_{n}\right\}$ generated by the above iterative scheme strongly converges to the unique solution of the variational inequality

$$
\left\langle(B-r f) x^{*}, x-x^{*}\right\rangle \geq 0, \quad x \in C
$$

which is the optimality condition for the minimization problem $\min _{x \in F(S)}(1 / 2)\langle B x, x\rangle-h(x)$, where $h$ is a potential function for $\delta f$ (i.e., $h^{\prime}(x)=\delta f(x)$ for $x \in H$ ).

Concerning a family of nonlinear mappings has been considered by many authors; see, for example, [9-21] and the references therein. The well-known convex feasibility problem reduces to finding a point in the intersection of the fixed point sets of a family of nonexpansive mappings. The problem of finding an optimal point that minimizes a given cost function over common set of fixed points of a family of nonexpansive mappings is of wide interdisciplinary interest and practical importance; see, for example, [16, 17].

Recently, Qin et al. [18] considered a general iterative algorithm for an infinite family of nonexpansive mapping in the framework of Hilbert spaces. To be more precise, they introduced the following general iterative algorithm:

$$
x_{0} \in C, \quad x_{n+1}=\lambda_{n} \gamma f\left(x_{n}\right)+\beta_{n} x_{n}+\left(\left(1-\beta_{n}\right) I-\lambda_{n} A\right) W_{n} x_{n}, \quad n \geq 0,
$$

where $f$ is a contraction on $H, A$ is a strongly positive bounded linear operator, $W_{n}$ are nonexpansive mappings which are generated by a finite family of nonexpansive mapping $T_{1}, T_{2}, \ldots$ as follows:

$$
\begin{aligned}
& U_{n, n+1}=I \text {, } \\
& U_{n, n}=\gamma_{n} T_{n} U_{n, n+1}+\left(1-\gamma_{n}\right) I \text {, } \\
& U_{n, n-1}=\gamma_{n-1} T_{n-1} U_{n, n}+\left(1-\gamma_{n-1}\right) I \text {, } \\
& \vdots \\
& U_{n, k}=\gamma_{k} T_{k} U_{n, k+1}+\left(1-\gamma_{k}\right) I \text {, } \\
& u_{n, k-1}=\gamma_{k-1} T_{k-1} U_{n, k}+\left(1-\gamma_{k-1}\right) I \text {, } \\
& U_{n, 2}=\gamma_{2} T_{2} U_{u, 3}+\left(1-\gamma_{2}\right) I, \\
& W_{n}=U_{n, 1}=\gamma_{1} T_{1} U_{n, 2}+\left(1-\gamma_{1}\right) I \text {, }
\end{aligned}
$$

where $\left\{\gamma_{1}\right\},\left\{\gamma_{2}\right\}, \ldots$ are real numbers such that $0 \leq \gamma \leq 1, T_{1}, T_{2}, \ldots$ become an infinite family of mappings of $C$ into itself. Nonexpansivity of each $T_{i}$ ensures the nonexpansivity of $W_{n}$. 
Concerning $W_{n}$ we have the following lemmas which are important to prove our main results.

Lemma 1.1 (see [19]). Let $C$ be a nonempty closed convex subset of a strictly convex Banach space E. Let $T_{1}, T_{2}, \ldots$ be nonexpansive mappings of $C$ into itself such that $\bigcap_{n=1}^{\infty} F\left(T_{n}\right)$ is nonempty, and let $\gamma_{1}, \gamma_{2}, \ldots$ be real numbers such that $0<\gamma_{n} \leq \eta<1$ for any $n \geq 1$. Then, for every $x \in C$ and $k \in N$, the limit $\lim _{n \rightarrow \infty} U_{n, k} x$ exists.

Using Lemma 1.1, one can define the mapping $W$ of $C$ into itself as follows. $W x=$ $\lim _{n \rightarrow \infty} W_{n} x=\lim _{n \rightarrow \infty} U_{n, 1} x$, for every $x \in C$. Such a $W$ is called the $W$-mapping generated by $T_{1}, T_{2}, \ldots$ and $\gamma_{1}, \gamma_{2}, \ldots$. Throughout this paper, we will assume that $0<\gamma_{n} \leq \eta<1$ for all $n \geq 1$.

Lemma 1.2 (see [19]). Let $C$ be a nonempty closed convex subset of a strictly convex Banach space $E$. Let $T_{1}, T_{2}, \ldots$ be nonexpansive mappings of $C$ into itself such that $\bigcap_{n=1}^{\infty} F\left(T_{n}\right)$ is nonempty, and let $\gamma_{1}, \gamma_{2}, \ldots$ be real numbers such that $0<\gamma_{n} \leq \eta<1$ for any $n \geq 1$. Then, $F(W)=\bigcap_{n=1}^{\infty} F\left(T_{n}\right)$.

Motivated by the above results, in this paper, we study the problem of approximating a common element in the common fixed point set of an infinite family of nonexpansive mappings, and in the solution set of a variational inequality involving an inversestrongly monotone mapping based on a viscosity approximation iterative method. Strong convergence theorems of common elements are established in the framework of Hilbert spaces.

In order to prove our main results, we need the following lemmas.

Lemma 1.3 (see [5]). Assume $B$ is a strongly positive linear bounded operator on a Hilbert space $H$ with coefficient $\bar{\gamma}>0$ and $0<\rho \leq\|B\|^{-1}$. Then $\|I-\rho B\| \leq 1-\rho \bar{\gamma}$.

Lemma 1.4 (see [22]). Assume that $\left\{\alpha_{n}\right\}$ is a sequence of nonnegative real numbers such that

$$
\alpha_{n+1} \leq\left(1-\gamma_{n}\right) \alpha_{n}+\delta_{n}
$$

where $\gamma_{n}$ is a sequence in $(0,1)$ and $\left\{\delta_{n}\right\}$ is a sequence such that

(i) $\sum_{n=1}^{\infty} \gamma_{n}=\infty$;

(ii) $\lim \sup _{n \rightarrow \infty} \delta_{n} / \gamma_{n} \leq 0$ or $\sum_{n=1}^{\infty}\left|\delta_{n}\right|<\infty$.

Then $\lim _{n \rightarrow \infty} \alpha_{n}=0$.

Lemma 1.5 (see [23]). Let $\left\{x_{n}\right\}$ and $\left\{y_{n}\right\}$ be bounded sequences in a Banach space $X$ and let $\left\{\beta_{n}\right\}$ be a sequence in $[0,1]$ with $0<\liminf _{n \rightarrow \infty} \beta_{n} \leq \limsup _{n \rightarrow \infty} \beta_{n}<1$. Suppose $x_{n+1}=\left(1-\beta_{n}\right) y_{n}+\beta_{n} x_{n}$ for all integers $n \geq 0$ and

$$
\limsup _{n \rightarrow \infty}\left(\left\|y_{n+1}-y_{n}\right\|-\left\|x_{n+1}-x_{n}\right\|\right) \leq 0
$$

Then $\lim _{n \rightarrow \infty}\left\|y_{n}-x_{n}\right\|=0$. 
Lemma 1.6 (see $[14,15])$. Let $K$ be a nonempty closed convex subset of a Hilbert space $H,\left\{T_{i}: C \rightarrow\right.$ C) be a family of infinitely nonexpansive mappings with $\bigcap_{i=1}^{\infty} F\left(T_{i}\right),\left\{\gamma_{n}\right\}$ be a real sequence such that $0<\gamma_{n} \leq b<1$ for each $n \geq 1$. If $C$ is any bounded subset of $K$, then $\lim _{n \rightarrow \infty} \sup _{x \in C}\left\|W x-W_{n} x\right\|=0$.

Lemma 1.7 (see [5]). Let $H$ be a Hilbert space. Let B be a strongly positive linear bounded selfadjoint operator with the constant $\bar{\gamma}>0$ and $f$ a contraction with the constant $\kappa$. Assume that $0<$ $\gamma<\bar{\gamma} / \kappa$. Let $T$ be a nonexpansive mapping with a fixed point $x_{t} \in H$ of the contraction $x \mapsto$ $t_{\gamma} f(x)+(I-t B) T x$. Then $\left\{x_{t}\right\}$ converges strongly as $t \rightarrow 0$ to a fixed point $\bar{x}$ of $T$, which solves the variational inequality

$$
\langle(A-\gamma f) \bar{x}, z-\bar{x}\rangle \leq 0, \quad \forall z \in F(T) .
$$

Equivalently, we have $P_{F(T)}(I-A+\gamma f) \bar{x}=\bar{x}$.

\section{Main Results}

Theorem 2.1. Let $H$ be a real Hilbert space and $C$ a nonempty closed convex subset of $H$. Let $A: C \rightarrow H$ be an $\alpha$-inverse-strongly monotone mapping and $f: C \rightarrow C$ a $\kappa$-contraction. Let $\left\{T_{i}\right\}_{i=1}^{\infty}$ be an infinite family of nonexpansive mappings from $C$ into itself such that $F:=$ $\bigcap_{i=1}^{\infty} F\left(T_{i}\right) \cap \operatorname{VI}(C, A) \neq \emptyset$. Let $B$ be a strongly positive linear bounded self-adjoint operator of $C$ into itself with the constant $\bar{\gamma}>0$. Let $\left\{x_{n}\right\}$ be a sequence generated in

$$
\begin{gathered}
x_{1} \in C, \\
y_{n}=\beta_{n} \gamma f\left(x_{n}\right)+\left(I-\beta_{n} B\right) W_{n} P_{C}\left(I-r_{n} A\right) x_{n}, \\
x_{n+1}=\alpha_{n} x_{n}+\left(1-\alpha_{n}\right) P_{C} y_{n}, \quad n \geq 1,
\end{gathered}
$$

where $W_{n}$ is generated in (1.18), $\left\{\alpha_{n}\right\}$ and $\left\{\beta_{n}\right\}$ are real number sequences in $(0,1)$. Assume that the control sequence $\left\{\alpha_{n}\right\},\left\{\beta_{n}\right\}$, and $\left\{r_{n}\right\}$ satisfy the following restrictions:

(i) $\lim _{n \rightarrow \infty} \beta_{n}=0, \sum_{n=1}^{\infty} \beta_{n}=\infty$;

(ii) $0<\liminf _{n \rightarrow \infty} \alpha_{n} \leq \limsup \sup _{n \rightarrow \infty} \alpha_{n}<1$;

(iii) $\lim _{n \rightarrow \infty}\left|r_{n+1}-r_{n}\right|=0$;

(iv) $\left\{r_{n}\right\} \subset[a, b]$ for some $a, b$ with $0<a<b<2 \alpha$.

Assume that $0<\gamma<\bar{\gamma} / \kappa$. Then $\left\{x_{n}\right\}$ strongly converges to some point $q$, where $q \in F$, where $q=P_{F}(\gamma f+(I-B))(q)$, which solves the variation inequality

$$
\langle\gamma f(q)-B q, p-q\rangle \leq 0, \quad \forall p \in F
$$


Proof. First, we show that the mapping $I-r_{n} A$ is nonexpansive. Notice that

$$
\begin{aligned}
\left\|\left(I-r_{n} A\right) x-\left(I-r_{n} A\right) y\right\|^{2} & =\left\|x-y-r_{n}(A x-A y)\right\|^{2} \\
& =\|x-y\|^{2}-2 r_{n}\langle x-y, A x-A y\rangle+r_{n}^{2}\|A x-A y\|^{2} \\
& \leq\|x-y\|^{2}+r_{n}\left(r_{n}-2 \alpha\right)\|A x-A y\|^{2} \\
& \leq\|x-y\|^{2}, \quad \forall x, y \in C,
\end{aligned}
$$

which implies that the mapping $I-r_{n} A$ is nonexpansive. Since the condition (i), we may assume, with no loss of generality, that $\beta_{n}<\|B\|^{-1}$ for all $n$. From Lemma 1.3, we know that if $0<\rho \leq\|B\|^{-1}$, then $\|I-\rho B\| \leq 1-\rho \bar{\gamma}$. Letting $p \in F$, we have

$$
\begin{aligned}
\left\|y_{n}-p\right\| & =\left\|\beta_{n}\left(\gamma f\left(x_{n}\right)-B p\right)+\left(I-\beta_{n} B\right)\left(W_{n} P_{C}\left(I-r_{n} A\right) x_{n}-p\right)\right\| \\
& \leq \beta_{n}\left\|\gamma f\left(x_{n}\right)-B p\right\|+\left(1-\beta_{n} \bar{\gamma}\right)\left\|W_{n} P_{C}\left(I-r_{n} A\right) x_{n}-p\right\| \\
& \leq \beta_{n} \gamma\left\|f\left(x_{n}\right)-f(p)\right\|+\beta_{n}\|\gamma f(p)-B p\|+\left(1-\beta_{n} \bar{\gamma}\right)\left\|x_{n}-p\right\| \\
& =\left[1-\beta_{n}(\bar{\gamma}-\kappa \gamma)\right]\left\|x_{n}-p\right\|+\beta_{n}\|\gamma f(p)-B p\| .
\end{aligned}
$$

On the other hand, we have

$$
\begin{aligned}
\left\|x_{n+1}-p\right\| & =\left\|\alpha_{n}\left(x_{n}-p\right)+\left(1-\alpha_{n}\right)\left(P_{C} y_{n}-p\right)\right\| \\
& \leq \alpha_{n}\left\|x_{n}-p\right\|+\left(1-\alpha_{n}\right)\left\|y_{n}-p\right\| \\
& \leq \alpha_{n}\left\|x_{n}-p\right\|+\left(1-\alpha_{n}\right)\left[\left(1-\beta_{n}(\bar{\gamma}-\gamma \kappa)\right)\left\|x_{n}-p\right\|+\beta_{n}\|\gamma f(p)-B p\|\right] .
\end{aligned}
$$

By simple induction, we have

$$
\left\|x_{n}-p\right\| \leq \max \left\{\left\|x_{0}-p\right\|, \frac{\|B p-\gamma f(p)\|}{\bar{\gamma}-\gamma \mathcal{K}}\right\},
$$

which gives that the sequence $\left\{x_{n}\right\}$ is bounded, so is $\left\{y_{n}\right\}$.

Next, we prove $\lim _{n \rightarrow \infty}\left|x_{n+1}-x_{n}\right| \mid=0$. Put $\rho_{n}=P_{C}\left(I-r_{n} A\right) x_{n}$. Next, we compute

$$
\begin{aligned}
\left\|\rho_{n}-\rho_{n+1}\right\| & =\left\|P_{C}\left(I-r_{n} A\right) x_{n}-P_{C}\left(I-r_{n+1} A\right) x_{n+1}\right\| \\
& \leq\left\|\left(I-r_{n} A\right) x_{n}-\left(I-r_{n+1} A\right) x_{n+1}\right\| \\
& =\left\|\left(x_{n}-r_{n} A x_{n}\right)-\left(x_{n+1}-r_{n} A x_{n+1}\right)+\left(r_{n+1}-r_{n}\right) A x_{n+1}\right\| \\
& \leq\left\|x_{n}-x_{n+1}\right\|+\left|r_{n+1}-r_{n}\right| M_{1},
\end{aligned}
$$


where $M_{1}$ is an appropriate constant such that $M_{1} \geq \sup _{n \geq 1}\left\{\left\|A x_{n}\right\|\right\}$. It follows that

$$
\begin{aligned}
\left\|y_{n}-y_{n+1}\right\|= & \|\left(I-\beta_{n+1} B\right)\left(W_{n+1} \rho_{n+1}-W_{n} \rho_{n}\right)-\left(\beta_{n+1}-\beta_{n}\right) B W_{n} \rho_{n} \\
& +\gamma\left[\beta_{n+1}\left(f\left(x_{n+1}\right)-f\left(x_{n}\right)\right)+f\left(x_{n}\right)\left(\beta_{n+1}-\beta_{n}\right)\right] \| \\
\leq & \left(1-\beta_{n+1} \bar{\gamma}\right)\left(\left\|\rho_{n+1}-\rho_{n}\right\|+\left\|W_{n+1} \rho_{n}-W_{n} \rho_{n}\right\|\right) \\
& +\left|\beta_{n+1}-\beta_{n}\right| M_{2}+\gamma \beta_{n+1} \kappa\left\|x_{n+1}-x_{n}\right\|,
\end{aligned}
$$

where $M_{2}$ is an appropriate constant such that

$$
M_{2} \geq \max \left\{\sup _{n \geq 1}\left\{\left\|B W_{n} \rho_{n}\right\|\right\}, \gamma \sup _{n \geq 1}\left\{\left\|f\left(x_{n}\right)\right\|\right\}\right\} .
$$

Since $T_{i}$ and $U_{n, i}$ are nonexpansive, we have from (1.18) that

$$
\begin{aligned}
\left\|W_{n+1} \rho_{n}-W_{n} \rho_{n}\right\| & =\left\|\gamma_{1} T_{1} U_{n+1,2} \rho_{n}-\gamma_{1} T_{1} U_{n, 2} \rho_{n}\right\| \\
& \leq \gamma_{1}\left\|U_{n+1,2} \rho-U_{n, 2} \rho_{n}\right\| \\
& =\gamma_{1}\left\|\gamma_{2} T_{2} U_{u+1,3} \rho_{n}-\gamma_{2} T_{2} U_{n, 3} \rho_{n}\right\| \\
& \leq \gamma_{1} \gamma_{2}\left\|U_{u+1,3} \rho_{n}-U_{n, 3} \rho_{n}\right\| \\
& \leq \cdots \\
& \leq \gamma_{1} \gamma_{2} \cdots \gamma_{n}\left\|U_{n+1, n+1} \rho_{n}-U_{n, n+1} \rho_{n}\right\| \\
& \leq M_{3} \prod_{i=1}^{n} \gamma_{i},
\end{aligned}
$$

where $M_{3} \geq 0$ is an appropriate constant such that $\left\|U_{n+1, n+1} \rho_{n}-U_{n, n+1} \rho_{n}\right\| \leq M_{3}$, for all $n \geq 0$. Substitute (2.7) and (2.10) into (2.8) yields that

$$
\begin{aligned}
\left\|y_{n}-y_{n+1}\right\| \leq & {\left[1-\beta_{n+1}(\bar{\gamma}-\kappa \gamma)\right]\left\|x_{n+1}-x_{n}\right\| } \\
& +M_{4}\left(\left|r_{n+1}-r_{n}\right|+\left|\beta_{n+1}-\beta_{n}\right|+\prod_{i=1}^{n} \gamma_{i}\right),
\end{aligned}
$$

where $M_{4}$ is an appropriate appropriate constant such that $M_{4} \geq \max \left\{M_{1}, M_{2}, M_{3}\right\}$. From the conditions (i) and (iii), we have

$$
\limsup _{n \rightarrow \infty}\left\{\left\|y_{n+1}-y_{n}\right\|-\left\|x_{n+1}-x_{n}\right\|\right\} \leq 0 .
$$

By virtue of Lemma 1.5, we obtain that

$$
\lim _{n \rightarrow \infty}\left\|y_{n}-x_{n}\right\|=0
$$


On the other hand, we have

$$
\left\|x_{n+1}-x_{n}\right\|=\left(1-\alpha_{n}\right)\left\|x_{n}-P_{C} y_{n}\right\| \leq\left\|x_{n}-y_{n}\right\| .
$$

This implies from (2.13) that

$$
\lim _{n \rightarrow \infty}\left\|x_{n+1}-x_{n}\right\|=0
$$

Next, we show $\lim _{n \rightarrow \infty}\left\|W \rho_{n}-\rho_{n}\right\|=0$. Observing that

$$
y_{n}-W_{n} \rho_{n}=\beta_{n}\left(\gamma f\left(x_{n}\right)-B W_{n} \rho_{n}\right)
$$

and the condition (i), we can easily get

$$
\lim _{n \rightarrow \infty}\left\|W_{n} \rho_{n}-y_{n}\right\|=0
$$

Notice that

$$
\begin{aligned}
\left\|\rho_{n}-p\right\|^{2} & =\left\|P_{C}\left(I-r_{n} A\right) x_{n}-P_{C}\left(I-r_{n} A\right) p\right\|^{2} \\
& \leq\left\|\left(x_{n}-p\right)-r_{n}\left(A x_{n}-A p\right)\right\|^{2} \\
& =\left\|x_{n}-p\right\|^{2}-2 r_{n}\left\langle x_{n}-p, A x_{n}-A p\right\rangle+r_{n}^{2}\left\|A x_{n}-A p\right\|^{2} \\
& \leq\left\|x_{n}-p\right\|^{2}-2 r_{n} \alpha\left\|A x_{n}-A p\right\|^{2}+r_{n}^{2}\left\|A x_{n}-A p\right\|^{2} \\
& =\left\|x_{n}-p\right\|^{2}-r_{n}\left(2 \alpha-r_{n}\right)\left\|A x_{n}-A p\right\|^{2} .
\end{aligned}
$$

On the other hand, we have

$$
\begin{aligned}
\left\|y_{n}-p\right\|^{2} & =\left\|\beta_{n}\left(\gamma f\left(x_{n}\right)-B p\right)+\left(I-\beta_{n} B\right)\left(W_{n} \rho_{n}-p\right)\right\|^{2} \\
& \leq\left(\beta_{n}\left\|\gamma f\left(x_{n}\right)-B p\right\|+\left(1-\beta_{n} \bar{\gamma}\right)\left\|\rho_{n}-p\right\|\right)^{2} \\
& \leq \beta_{n}\left\|\gamma f\left(x_{n}\right)-B p\right\|^{2}+\left\|\rho_{n}-p\right\|^{2}+2 \beta_{n}\left\|\gamma f\left(x_{n}\right)-B p\right\|\left\|\rho_{n}-p\right\|
\end{aligned}
$$

from which it follows that

$$
\begin{aligned}
\left\|x_{n+1}-p\right\|^{2}= & \left\|\alpha_{n}\left(x_{n}-p\right)+\left(1-\alpha_{n}\right)\left(P_{C} y_{n}-p\right)\right\|^{2} \\
\leq & \alpha_{n}\left\|x_{n}-p\right\|^{2}+\left(1-\alpha_{n}\right)\left\|y_{n}-p\right\|^{2} \\
\leq & \alpha_{n}\left\|x_{n}-p\right\|^{2}+\left(1-\alpha_{n}\right) \\
& \times\left[\beta_{n}\left\|\gamma f\left(x_{n}\right)-B p\right\|^{2}+\left\|\rho_{n}-p\right\|^{2}+2 \beta_{n}\left\|\gamma f\left(x_{n}\right)-B p\right\|\left\|\rho_{n}-p\right\|\right] .
\end{aligned}
$$


Substituting (2.18) into (2.20), we arrive at

$$
\begin{aligned}
\left\|x_{n+1}-p\right\|^{2} \leq & \left\|x_{n}-p\right\|^{2}+\beta_{n}\left\|\gamma f\left(x_{n}\right)-B p\right\|^{2} \\
& -\left(1-\alpha_{n}\right) r_{n}\left(2 \alpha-r_{n}\right)\left\|A x_{n}-A p\right\|^{2} \\
& +2 \beta_{n}\left\|\gamma f\left(x_{n}\right)-B p\right\|\left\|\rho_{n}-p\right\| .
\end{aligned}
$$

It follows that

$$
\begin{aligned}
& \left(1-\alpha_{n}\right) r_{n}\left(2 \alpha-r_{n}\right)\left\|A x_{n}-A p\right\|^{2} \\
& \quad \leq \beta_{n}\left\|\gamma f\left(x_{n}\right)-B p\right\|^{2}+\left\|x_{n}-p\right\|^{2}-\left\|x_{n+1}-p\right\|^{2}+2 \beta_{n}\left\|\gamma f\left(x_{n}\right)-B p\right\|\left\|\rho_{n}-p\right\| \\
& \quad \leq \beta_{n}\left\|\gamma f\left(x_{n}\right)-p\right\|^{2}+\left(\left\|x_{n}-p\right\|+\left\|x_{n+1}-p\right\|\right)\left\|x_{n}-x_{n+1}\right\|+2 \beta_{n}\left\|\gamma f\left(x_{n}\right)-B p\right\|\left\|\rho_{n}-p\right\| .
\end{aligned}
$$

In view of the restrictions (i), and (iv), we find from (2.15) that

$$
\lim _{n \rightarrow \infty}\left\|A x_{n}-A p\right\|=0 .
$$

Observe that

$$
\begin{aligned}
\left\|\rho_{n}-p\right\|^{2}= & \left\|P_{C}\left(I-r_{n} A\right) x_{n}-P_{C}\left(I-r_{n} A\right) p\right\|^{2} \\
\leq & \left\langle\left(I-r_{n} A\right) x_{n}-\left(I-r_{n} A\right) p, \rho_{n}-p\right\rangle \\
= & \frac{1}{2}\left\{\left\|\left(I-r_{n} A\right) x_{n}-\left(I-r_{n} A\right) p\right\|^{2}+\left\|\rho_{n}-p\right\|^{2}\right. \\
& \left.\quad-\left\|\left(I-r_{n} A\right) x_{n}-\left(I-r_{n} A\right) p-\left(\rho_{n}-p\right)\right\|^{2}\right\} \\
\leq & \frac{1}{2}\left\{\left\|x_{n}-p\right\|^{2}+\left\|\rho_{n}-p\right\|^{2}-\left\|\left(x_{n}-\rho_{n}\right)-r_{n}\left(A x_{n}-A p\right)\right\|^{2}\right\} \\
= & \frac{1}{2}\left\{\left\|x_{n}-p\right\|^{2}+\left\|\rho_{n}-p\right\|^{2}-\left\|x_{n}-\rho_{n}\right\|^{2}-r_{n}^{2}\left\|A x_{n}-A p\right\|^{2}\right. \\
& \left.\quad+2 r_{n}\left\langle x_{n}-\rho_{n}, A x_{n}-A p\right\rangle\right\},
\end{aligned}
$$

which yields that

$$
\left\|\rho_{n}-p\right\|^{2} \leq\left\|x_{n}-p\right\|^{2}-\left\|\rho_{n}-x_{n}\right\|^{2}+2 r_{n}\left\|\rho_{n}-x_{n}\right\|\left\|A x_{n}-A p\right\| .
$$

Substituting (2.25) into (2.20), we have

$$
\begin{aligned}
\left\|x_{n+1}-p\right\|^{2} \leq & \left\|x_{n}-p\right\|^{2}+\beta_{n}\left\|\gamma f\left(x_{n}\right)-B p\right\|^{2}+2 r_{n}\left\|\rho_{n}-x_{n}\right\|\left\|A x_{n}-A p\right\| \\
& -\left(1-\alpha_{n}\right)\left\|\rho_{n}-x_{n}\right\|^{2}+2 \beta_{n}\left\|\gamma f\left(x_{n}\right)-B p\right\|\left\|\rho_{n}-p\right\| .
\end{aligned}
$$


This implies that

$$
\begin{aligned}
(1- & \left.\alpha_{n}\right)\left\|\rho_{n}-x_{n}\right\|^{2} \\
\leq & \left\|x_{n}-p\right\|^{2}-\left\|x_{n+1}-p\right\|^{2}+\beta_{n}\left\|\gamma f\left(x_{n}\right)-B p\right\|^{2}+2 r_{n}\left\|\rho_{n}-x_{n}\right\|\left\|A x_{n}-A p\right\| \\
& +2 \beta_{n}\left\|\gamma f\left(x_{n}\right)-B p\right\|\left\|\rho_{n}-p\right\| \\
\leq & \left(\left\|x_{n}-p\right\|+\left\|x_{n+1}-p\right\|\right)\left\|x_{n}-x_{n+1}\right\|+\beta_{n}\left\|\gamma f\left(x_{n}\right)-B p\right\|^{2} \\
& +2 r_{n}\left\|\rho_{n}-x_{n}\right\|\left\|A x_{n}-A p\right\|+2 \beta_{n}\left\|\gamma f\left(x_{n}\right)-B p\right\|\left\|\rho_{n}-p\right\| .
\end{aligned}
$$

In view of the restrictions (i) and (ii), we find from (2.15) and (2.23) that

$$
\lim _{n \rightarrow \infty}\left\|\rho_{n}-x_{n}\right\|=0 .
$$

On the other hand, we have

$$
\left\|\rho_{n}-W_{n} \rho_{n}\right\| \leq\left\|x_{n}-\rho_{n}\right\|+\left\|x_{n}-y_{n}\right\|+\left\|y_{n}-W_{n} \rho_{n}\right\| .
$$

It follows from (2.13), (2.17) and (2.28) that $\lim _{n \rightarrow \infty}\left\|W_{n} \rho_{n}-\rho_{n}\right\|=0$. From Lemma 1.6, we find that $\left\|W \rho_{n}-W_{n} \rho_{n}\right\| \rightarrow 0$ as $n \rightarrow \infty$. Notice that

$$
\left\|W \rho_{n}-\rho_{n}\right\| \leq\left\|W_{n} \rho_{n}-\rho_{n}\right\|+\left\|W_{n} \rho_{n}-W \rho_{n}\right\|,
$$

from which it follows that

$$
\lim _{n \rightarrow \infty}\left\|W \rho_{n}-\rho_{n}\right\|=0
$$

Next, we show $\lim \sup _{n \rightarrow \infty}\left\langle\gamma f(q)-B q, x_{n}-q\right\rangle \leq 0$, where $q=P_{F}(\gamma f+(I-B))(q)$. To show it, we choose a subsequence $\left\{x_{n_{i}}\right\}$ of $\left\{x_{n}\right\}$ such that

$$
\limsup _{n \rightarrow \infty}\left\langle\gamma f(q)-B q, x_{n}-q\right\rangle=\lim _{i \rightarrow \infty}\left\langle\gamma f(q)-B q, x_{n_{i}}-q\right\rangle
$$

As $\left\{x_{n_{i}}\right\}$ is bounded, we have that there is a subsequence $\left\{x_{n_{i}}\right\}$ of $\left\{x_{n_{i}}\right\}$ converges weakly to $p$. We may assume, without loss of generality, that $x_{n_{i}} \rightarrow p$. Hence we have $p \in F$. Indeed, let us first show that $p \in \operatorname{VI}(C, A)$. Put

$$
T w_{1}= \begin{cases}A w_{1}+N_{C} w_{1}, & w_{1} \in C, \\ \emptyset, & w_{1} \notin C .\end{cases}
$$

Since $A$ is inverse-strongly monotone, we see that $T$ is maximal monotone. Let $\left(w_{1}, w_{2}\right) \in$ $G(T)$. Since $w_{2}-A w_{1} \in N_{C} w_{1}$ and $\rho_{n} \in C$, we have

$$
\left\langle w_{1}-\rho_{n}, w_{2}-A w_{1}\right\rangle \geq 0
$$


On the other hand, from $\rho_{n}=P_{C}\left(I-r_{n} A\right) x_{n}$, we have

$$
\left\langle w_{1}-\rho_{n}, \rho_{n}-\left(I-r_{n} A\right) x_{n}\right\rangle \geq 0
$$

and hence

$$
\left\langle w_{1}-\rho_{n}, \frac{\rho_{n}-x_{n}}{r_{n}}+A x_{n}\right\rangle \geq 0
$$

It follows that

$$
\begin{aligned}
\left\langle w_{1}-\rho_{n_{i}}, w_{2}\right\rangle \geq & \left\langle w_{1}-\rho_{n_{i}}, A w_{1}\right\rangle \\
\geq & \left\langle w_{1}-\rho_{n_{i}}, A w_{1}\right\rangle \\
& -\left\langle w_{1}-\rho_{n_{i}}, \frac{\rho_{n_{i}}-x_{n_{i}}}{r_{n_{i}}}+A x_{n_{i}}\right\rangle \\
\geq & \left\langle w_{1}-\rho_{n_{i}}, A w_{1}-\frac{\rho_{n_{i}}-x_{n_{i}}}{r_{n_{i}}}-A x_{n_{i}}\right\rangle \\
= & \left\langle w_{1}-\rho_{n_{i}}, A w_{1}-A \rho_{n_{i}}\right\rangle+\left\langle w_{1}-\rho_{n_{i}}, A \rho_{n_{i}}-A x_{n_{i}}\right\rangle \\
& -\left\langle w_{1}-\rho_{n_{i}}, \frac{\rho_{n_{i}}-x_{n_{i}}}{r_{n_{i}}}\right\rangle \\
\geq & \left\langle w_{1}-\rho_{n_{i}}, A \rho_{n_{i}}-A x_{n_{i}}\right\rangle-\left\langle w_{1}-\rho_{n_{i}}, \frac{\rho_{n_{i}}-x_{n_{i}}}{r_{n_{i}}}\right\rangle,
\end{aligned}
$$

which implies from (2.28) that $\left\langle w_{1}-p, w_{2}\right\rangle \geq 0$. We have $p \in T^{-1} 0$ and hence $p \in \operatorname{VI}(C, A)$. Next, let us show $p \in \bigcap_{i=1}^{\infty} F\left(T_{i}\right)$. Since Hilbert spaces are Opial's spaces, from (2.31), we have

$$
\begin{aligned}
\liminf _{i \rightarrow \infty}\left\|\rho_{n_{i}}-p\right\| & <\liminf _{i \rightarrow \infty}\left\|\rho_{n_{i}}-W p\right\| \\
& =\liminf _{i \rightarrow \infty}\left\|\rho_{n_{i}}-W \rho_{n_{i}}+W_{n} \rho_{n_{i}}-W p\right\| \\
& \leq \liminf _{i \rightarrow \infty}\left\|W \rho_{n_{i}}-W p\right\| \\
& \leq \liminf _{i \rightarrow \infty}\left\|\rho_{n_{i}}-p\right\|,
\end{aligned}
$$

which derives a contradiction. Thus, we have from Lemma 1.2 that $p \in F(W)=\bigcap_{i=1}^{\infty} F\left(T_{i}\right)$. On the other hand, we have

$$
\begin{aligned}
\limsup _{n \rightarrow \infty}\left\langle\gamma f(q)-B q, x_{n}-q\right\rangle & =\lim _{n \rightarrow \infty}\left\langle\gamma f(q)-B q, x_{n_{i}}-q\right\rangle \\
& =\langle\gamma f(q)-B q, p-q\rangle \\
& \leq 0 .
\end{aligned}
$$


Finally, we show $x_{n} \rightarrow q$ strongly as $n \rightarrow \infty$. Notice that

$$
\begin{aligned}
\left\|y_{n}-q\right\|^{2}= & \left\|\beta_{n}\left(\gamma f\left(x_{n}\right)-B q\right)+\left(I-\beta_{n} B\right)\left(W_{n} \rho_{n}-q\right)\right\|^{2} \\
\leq & \left(1-\beta_{n} \bar{\gamma}\right)^{2}\left\|W_{n} \rho_{n}-q\right\|^{2}+2 \beta_{n}\left\langle\gamma f\left(x_{n}\right)-B q, y_{n}-q\right\rangle \\
\leq & \left(1-\beta_{n} \bar{\gamma}\right)^{2}\left\|x_{n}-q\right\|^{2}+\kappa \gamma \beta_{n}\left(\left\|x_{n}-q\right\|^{2}+\left\|y_{n}-q\right\|^{2}\right) \\
& +2 \beta_{n}\left\langle\gamma f(q)-B q, y_{n}-q\right\rangle .
\end{aligned}
$$

Therefore, we have

$$
\begin{aligned}
\left\|y_{n}-q\right\|^{2} \leq & \frac{\left(1-\beta_{n} \bar{\gamma}\right)^{2}+\beta_{n} \gamma \mathcal{k}}{1-\beta_{n} \gamma \mathcal{K}}\left\|x_{n}-q\right\|^{2}+\frac{2 \beta_{n}}{1-\alpha_{n} \gamma \kappa}\left\langle\gamma f(q)-B q, y_{n}-q\right\rangle \\
= & \frac{\left(1-2 \beta_{n} \bar{\gamma}+\beta_{n} \kappa \gamma\right)}{1-\beta_{n} \gamma \mathcal{K}}\left\|x_{n}-q\right\|^{2}+\frac{\beta_{n}^{2} \bar{\gamma}^{2}}{1-\beta_{n} \gamma \kappa}\left\|x_{n}-q\right\|^{2} \\
& +\frac{2 \beta_{n}}{1-\beta_{n} \gamma \kappa}\left\langle\gamma f(q)-B q, y_{n}-q\right\rangle \\
\leq & {\left[1-\frac{2 \beta_{n}(\bar{\gamma}-\kappa \gamma)}{1-\beta_{n} \gamma \kappa}\right]\left\|x_{n}-q\right\|^{2} } \\
& +\frac{2 \beta_{n}(\bar{\gamma}-\kappa \gamma)}{1-\beta_{n} \gamma \kappa}\left[\frac{1}{\bar{\gamma}-\kappa \gamma}\left\langle\gamma f(q)-B q, y_{n}-q\right\rangle+\frac{\alpha_{n} \bar{\gamma}^{2}}{2(\bar{\gamma}-\kappa \gamma)} M_{5}\right],
\end{aligned}
$$

where $M_{5}$ is an appropriate constant. On the other hand, we have

$$
\begin{aligned}
\left\|x_{n+1}-p\right\|^{2} & =\left\|\alpha_{n}\left(x_{n}-p\right)+\left(1-\alpha_{n}\right)\left(P_{C} y_{n}-p\right)\right\|^{2} \\
& \leq \alpha_{n}\left\|x_{n}-p\right\|^{2}+\left(1-\alpha_{n}\right)\left\|P_{C} y_{n}-p\right\|^{2} \\
& \leq \alpha_{n}\left\|x_{n}-p\right\|^{2}+\left(1-\alpha_{n}\right)\left\|y_{n}-p\right\|^{2} .
\end{aligned}
$$

Substitute (2.41) into (2.42) yields that

$$
\begin{aligned}
\left\|x_{n+1}-p\right\|^{2} \leq & {\left[1-\left(1-\alpha_{n}\right) \frac{2 \beta_{n}(\bar{\gamma}-\alpha \gamma)}{1-\beta_{n} \gamma \alpha}\right]\left\|x_{n}-q\right\|^{2} } \\
& +\left(1-\alpha_{n}\right) \frac{2 \beta_{n}(\bar{\gamma}-\alpha \gamma)}{1-\beta_{n} \gamma \alpha}\left[\frac{1}{\bar{\gamma}-\alpha \gamma}\left\langle\gamma f(q)-B q, y_{n}-q\right\rangle+\frac{\beta_{n} \bar{\gamma}^{2}}{2(\bar{\gamma}-\alpha \gamma)} M_{5}\right] .
\end{aligned}
$$


Put $l_{n}=\left(1-\alpha_{n}\right)\left(2 \beta_{n}\left(\bar{\gamma}-\alpha_{n} \gamma\right) /\left(1-\beta_{n} \alpha \gamma\right)\right)$ and

$$
t_{n}=\frac{1}{\bar{\gamma}-\alpha \gamma}\left\langle\gamma f(q)-B q, y_{n}-q\right\rangle+\frac{\beta_{n} \bar{\gamma}^{2}}{2(\bar{\gamma}-\alpha \gamma)} M_{5}
$$

That is,

$$
\left\|x_{n+1}-q\right\|^{2} \leq\left(1-l_{n}\right)\left\|x_{n}-q\right\|^{2}+l_{n} t_{n}
$$

Notice that

$$
\begin{aligned}
\left\langle\gamma f(q)-B q, y_{n}-q\right\rangle & =\left\langle\gamma f(q)-B q, y_{n}-x_{n}\right\rangle+\left\langle\gamma f(q)-B q, x_{n}-q\right\rangle \\
& \leq\|\gamma f(q)-B q\|\left\|y_{n}-x_{n}\right\|+\left\langle\gamma f(q)-B q, x_{n}-q\right\rangle .
\end{aligned}
$$

From (2.13) and (2.39) that

$$
\limsup _{n \rightarrow \infty}\left\langle\gamma f(q)-A q, y_{n}-q\right\rangle \leq 0
$$

It follows from the condition (i) and (2.47) that

$$
\lim _{n \rightarrow \infty} l_{n}=0, \quad \sum_{n=1}^{\infty} l_{n}=\infty, \quad \limsup _{n \rightarrow \infty} t_{n} \leq 0 .
$$

Apply Lemma 1.4 to (2.45) to conclude $x_{n} \rightarrow q$ as $n \rightarrow \infty$. This completes the proof.

For a single nonexpansive mapping, we have from Theorem 2.1 the following.

Corollary 2.2. Let $H$ be a real Hilbert space and $C$ a nonempty closed convex subset of $H$. Let $A: C \rightarrow H$ be an $\alpha$-inverse-strongly monotone mapping and $f: C \rightarrow C$ a $\kappa$-contraction. Let $T$ be a nonexpansive mapping from $C$ into itself such that $F:=F(T) \cap \operatorname{VI}(C, A) \neq \emptyset$. Let $B$ be a strongly positive linear bounded self-adjoint operator of $C$ into itself with the constant $\bar{\gamma}>0$. Let $\left\{x_{n}\right\}$ be a sequence generated in

$$
\begin{gathered}
x_{1} \in C, \\
y_{n}=\beta_{n} \gamma f\left(x_{n}\right)+\left(I-\beta_{n} B\right) T P_{C}\left(I-r_{n} A\right) x_{n}, \\
x_{n+1}=\alpha_{n} x_{n}+\left(1-\alpha_{n}\right) P_{C} y_{n}, \quad n \geq 1
\end{gathered}
$$

where $\left\{\alpha_{n}\right\}$ and $\left\{\beta_{n}\right\}$ are real number sequences in $(0,1)$. Assume that the control sequence $\left\{\alpha_{n}\right\}$, $\left\{\beta_{n}\right\}$ and $\left\{r_{n}\right\}$ satisfy the following restrictions:

(i) $\lim _{n \rightarrow \infty} \beta_{n}=0, \sum_{n=1}^{\infty} \beta_{n}=\infty$;

(ii) $0<\liminf _{n \rightarrow \infty} \alpha_{n} \leq \limsup _{n \rightarrow \infty} \alpha_{n}<1$; 
(iii) $\lim _{n \rightarrow \infty}\left|r_{n+1}-r_{n}\right|=0$;

(iv) $\left\{r_{n}\right\} \subset[a, b]$ for some $a, b$ with $0<a<b<2 \alpha$.

Assume that $0<\gamma<\bar{\gamma} / \kappa$. Then $\left\{x_{n}\right\}$ strongly converges to some point $q$, where $q \in F$, where $q=P_{F}(\gamma f+(I-B))(q)$, which solves the variation inequality

$$
\langle\gamma f(q)-B q, p-q\rangle \leq 0, \quad \forall p \in F
$$

Corollary 2.3. Let $H$ be a real Hilbert space and $C$ a nonempty closed convex subset of $H$. Let $f: C \rightarrow C$ be a $\mathcal{\kappa}$-contraction. Let $T$ be a nonexpansive mapping from $C$ into itself such that $F(T) \neq \emptyset$. Let $B$ be a strongly positive linear bounded self-adjoint operator of $C$ into itself with the constant $\bar{\gamma}>0$. Let $\left\{x_{n}\right\}$ be a sequence generated in

$$
\begin{gathered}
x_{1} \in C, \\
y_{n}=\beta_{n} \gamma f\left(x_{n}\right)+\left(I-\beta_{n} B\right) T x_{n}, \\
x_{n+1}=\alpha_{n} x_{n}+\left(1-\alpha_{n}\right) P_{C} y_{n}, \quad n \geq 1,
\end{gathered}
$$

where $\left\{\alpha_{n}\right\}$ and $\left\{\beta_{n}\right\}$ are real number sequences in $(0,1)$. Assume that the control sequence $\left\{\alpha_{n}\right\}$, and $\left\{\beta_{n}\right\}$ satisfy the following restrictions:

(i) $\lim _{n \rightarrow \infty} \beta_{n}=0, \sum_{n=1}^{\infty} \beta_{n}=\infty$;

(ii) $0<\liminf _{n \rightarrow \infty} \alpha_{n} \leq \limsup _{n \rightarrow \infty} \alpha_{n}<1$.

Assume that $0<\gamma<\bar{\gamma} / \mathcal{K}$. Then $\left\{x_{n}\right\}$ strongly converges to some point $q$, where $q \in F(T)$, where $q=P_{F}(\gamma f+(I-B))(q)$, which solves the variation inequality

$$
\langle\gamma f(q)-B q, p-q\rangle \leq 0, \quad \forall p \in F(T)
$$

If $B$ is the identity mapping, then Theorem 2.1 is reduced to the following.

Corollary 2.4. Let $H$ be a real Hilbert space and $C$ a nonempty closed convex subset of $H$. Let $A: C \rightarrow H$ be an $\alpha$-inverse-strongly monotone mapping and $f: C \rightarrow C$ a $\kappa$-contraction. Let $\left\{T_{i}\right\}_{i=1}^{\infty}$ be an infinite family of nonexpansive mappings from $C$ into itself such that $F:=\bigcap_{i=1}^{\infty} F\left(T_{i}\right) \cap$ $\mathrm{VI}(\mathrm{C}, \mathrm{A}) \neq \emptyset$. Let $\left\{x_{n}\right\}$ be a sequence generated in

$$
\begin{gathered}
x_{1} \in C, \\
y_{n}=\beta_{n} f\left(x_{n}\right)+\left(1-\beta_{n}\right) W_{n} P_{C}\left(I-r_{n} A\right) x_{n}, \\
x_{n+1}=\alpha_{n} x_{n}+\left(1-\alpha_{n}\right) y_{n}, \quad n \geq 1,
\end{gathered}
$$

where $W_{n}$ is generated in (1.18), $\left\{\alpha_{n}\right\}$ and $\left\{\beta_{n}\right\}$ are real number sequences in $(0,1)$. Assume that the control sequence $\left\{\alpha_{n}\right\},\left\{\beta_{n}\right\}$, and $\left\{r_{n}\right\}$ satisfy the following restrictions:

(i) $\lim _{n \rightarrow \infty} \beta_{n}=0, \sum_{n=1}^{\infty} \beta_{n}=\infty$;

(ii) $0<\liminf _{n \rightarrow \infty} \alpha_{n} \leq \limsup _{n \rightarrow \infty} \alpha_{n}<1$; 
(iii) $\lim _{n \rightarrow \infty}\left|r_{n+1}-r_{n}\right|=0$;

(iv) $\left\{r_{n}\right\} \subset[a, b]$ for some $a, b$ with $0<a<b<2 \alpha$.

Then $\left\{x_{n}\right\}$ strongly converges to some point $q$, where $q \in F$, where $q=P_{F} f(q)$, which solves the variation inequality

$$
\langle f(q)-q, p-q\rangle \leq 0, \quad \forall p \in F .
$$

\section{References}

[1] R. T. Rockafellar, "On the maximality of sums of nonlinear monotone operators," Transactions of the American Mathematical Society, vol. 149, pp. 75-88, 1970.

[2] W. Takahashi and M. Toyoda, "Weak convergence theorems for nonexpansive mappings and monotone mappings," Journal of Optimization Theory and Applications, vol. 118, no. 2, pp. 417-428, 2003.

[3] H. Iiduka and W. Takahashi, "Strong convergence theorems for nonexpansive mappings and inversestrongly monotone mappings," Nonlinear Analysis. Theory, Methods E Applications, vol. 61, no. 3, pp. 341-350, 2005.

[4] H. K. Xu, "An iterative approach to quadratic optimization," Journal of Optimization Theory and Applications, vol. 116, no. 3, pp. 659-678, 2003.

[5] G. Marino and H.-K. Xu, "A general iterative method for nonexpansive mappings in Hilbert spaces," Journal of Mathematical Analysis and Applications, vol. 318, no. 1, pp. 43-52, 2006.

[6] X. Qin, S. Y. Cho, and S. M. Kang, "On hybrid projection methods for asymptotically quasi- $\varphi$ nonexpansive mappings," Applied Mathematics and Computation, vol. 215, no. 11, pp. 3874-3883, 2010.

[7] F. Deutsch and I. Yamada, "Minimizing certain convex functions over the intersection of the fixed point sets of nonexpansive mappings," Numerical Functional Analysis and Optimization, vol. 19, no. 1-2, pp. 33-56, 1998.

[8] S. Y. Cho and S. M. Kang, "Approximation of fixed points of pseudocontraction semigroups based on a viscosity iterative process," Applied Mathematics Letters, vol. 24, no. 2, pp. 224-228, 2011.

[9] S. Y. Cho and S. M. Kang, "Approximation of common solutions of variational inequalities via strict pseudocontractions," Acta Mathematica Scientia Series B, vol. 32, no. 4, pp. 1607-1618, 2012.

[10] S. Y. Cho, X. Qin, and S. M. Kang, "Hybrid projection algorithms for treating common fixed points of a family of demicontinuous pseudocontractions," Applied Mathematics Letters, vol. 25, no. 5, pp. 854-857, 2012.

[11] X. Qin, S.-s. Chang, and Y. J. Cho, "Iterative methods for generalized equilibrium problems and fixed point problems with applications," Nonlinear Analysis. Real World Applications, vol. 11, no. 4, pp. 2963 2972, 2010.

[12] A. P. Chouhan and A. S. Ranadive, "Absorbing maps and common fixed point theorem in Menger space," Advances in Fixed Point Theory, vol. 2, pp. 108-119, 2012.

[13] J. Ye and J. Huang, "Strong convergence theorems for fixed point problems and generalized equilibrium problems of three relatively quasi-nonexpansive mappings in Banach spaces," Journal of Mathematical and Computational Science, vol. 1, no. 1, pp. 1-18, 2011.

[14] Z. Kadelburg and S. Radenovic, "Coupled fixed point results under tvs-cone metric and w-conedistance," Advances in Fixed Point Theory, vol. 2, pp. 29-46, 2012.

[15] Y. J. Cho, X. Qin, and S. M. Kang, "Some results for equilibrium problems and fixed point problems in Hilbert spaces," Journal of Computational Analysis and Applications, vol. 11, no. 2, pp. 287-294, 2009.

[16] F. Deutsch and H. Hundal, "The rate of convergence of Dykstra's cyclic projections algorithm: the polyhedral case," Numerical Functional Analysis and Optimization, vol. 15, no. 5-6, pp. 537-565, 1994.

[17] H. H. Bauschke, "The approximation of fixed points of compositions of nonexpansive mappings in Hilbert space," Journal of Mathematical Analysis and Applications, vol. 202, no. 1, pp. 150-159, 1996.

[18] X. Qin, M. Shang, and Y. Su, "Strong convergence of a general iterative algorithm for equilibrium problems and variational inequality problems," Mathematical and Computer Modelling, vol. 48, no. 7-8, pp. 1033-1046, 2008. 
[19] K. Shimoji and W. Takahashi, "Strong convergence to common fixed points of infinite nonexpansive mappings and applications," Taiwanese Journal of Mathematics, vol. 5, no. 2, pp. 387-404, 2001.

[20] R. Bhardwaj, "Some fixed point theorems in polish space using new type of contractive conditions," Advances in Fixed Point Theory, vol. 2, pp. 313-325, 2012.

[21] X. Qin, Y. J. Cho, J. I. Kang, and S. M. Kang, "Strong convergence theorems for an infinite family of nonexpansive mappings in Banach spaces," Journal of Computational and Applied Mathematics, vol. 230, no. 1, pp. 121-127, 2009.

[22] H. K. Xu, "An iterative approach to quadratic optimization," Journal of Optimization Theory and Applications, vol. 116, no. 3, pp. 659-678, 2003.

[23] T. Suzuki, "Strong convergence of Krasnoselskii and Mann's type sequences for one-parameter nonexpansive semigroups without Bochner integrals," Journal of Mathematical Analysis and Applications, vol. 305, no. 1, pp. 227-239, 2005. 


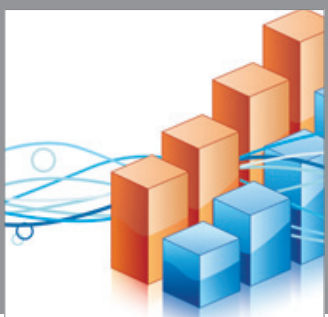

Advances in

Operations Research

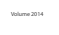

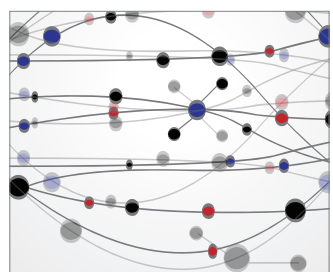

\section{The Scientific} World Journal
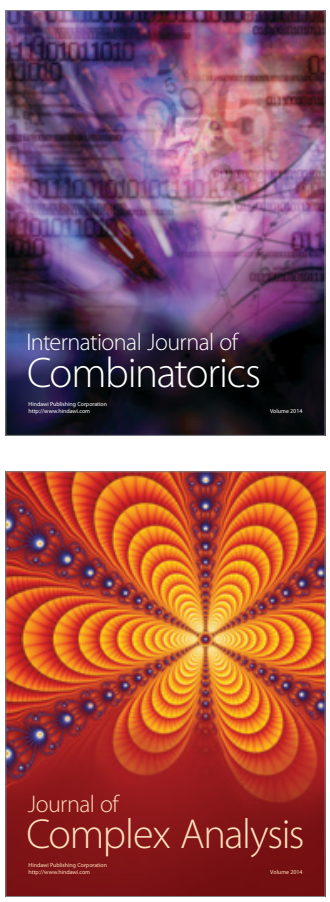

International Journal of

Mathematics and

Mathematical

Sciences
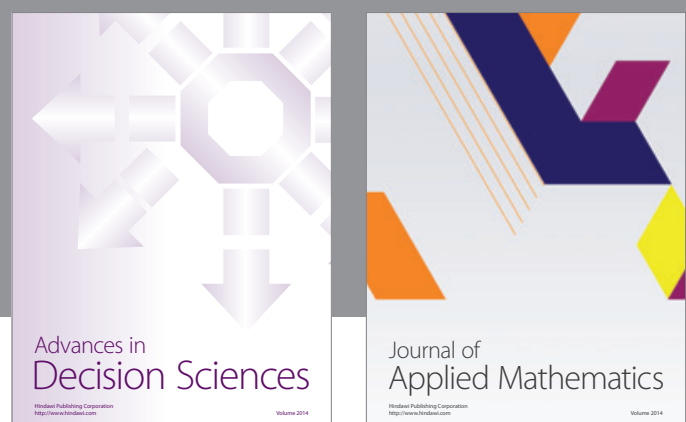

Journal of

Applied Mathematics
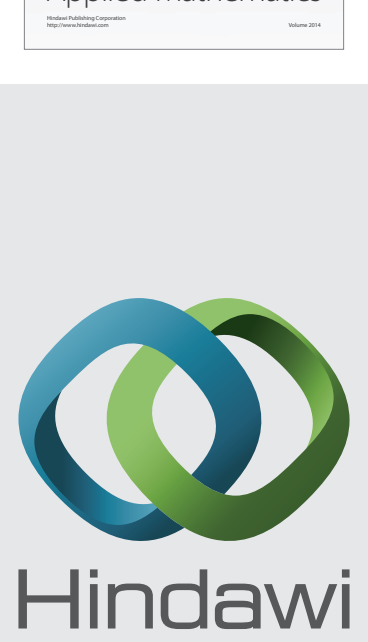

Submit your manuscripts at http://www.hindawi.com
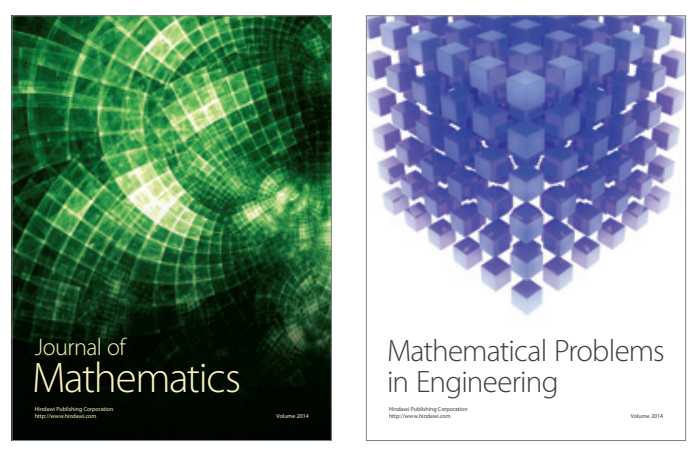

Mathematical Problems in Engineering
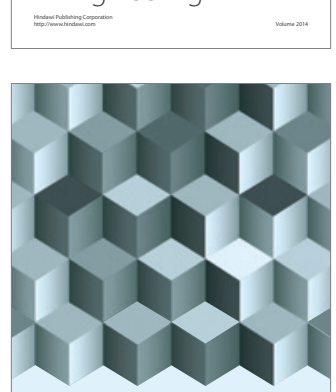

Journal of

Function Spaces
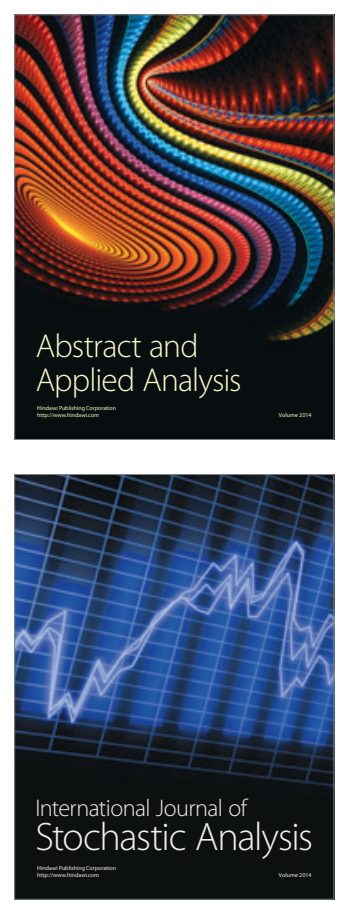

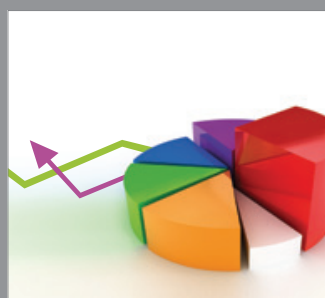

ournal of

Probability and Statistics

Promensencen
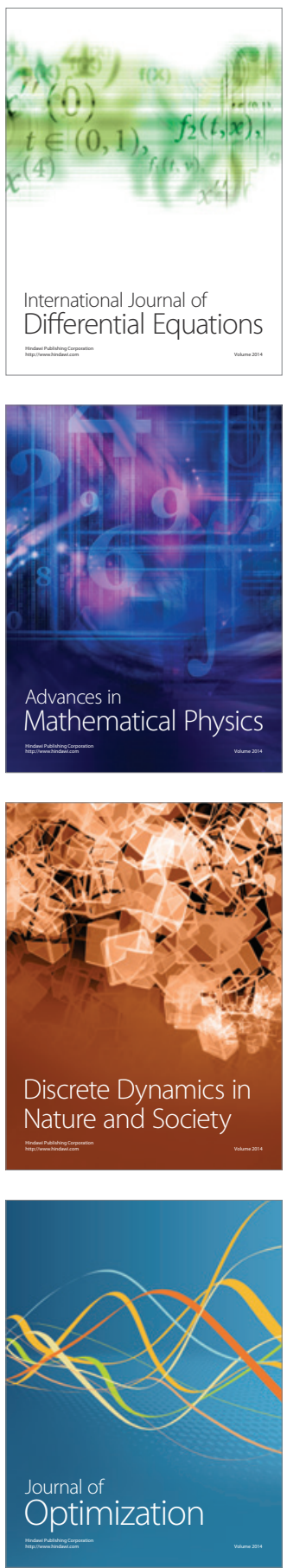